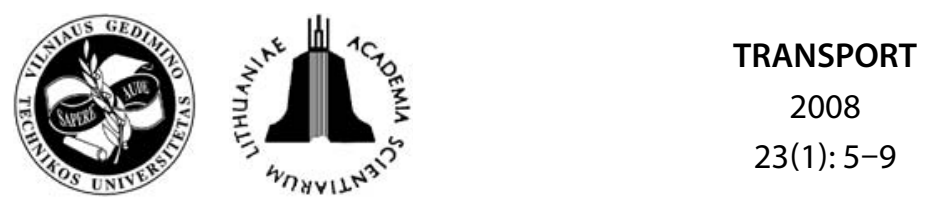

\title{
MODEL FOR DISTRIBUTION OF WAREHOUSES IN THE COMMERCIAL NETWORK IN OPTIMISING TRANSPORTATION OF GOODS
}

\author{
Adolfas Baublys \\ Transport Research Institute, Vilnius Gediminas Technical University, \\ Plytinès g. 27, LT-10105 Vilnius, Lithuania.E-mail: Adolfas.Baublys@ti.vgtu.lt
}

Received 6 January 2007; accepted 20 November 2007

\begin{abstract}
A model for distribution of warehouses in the commercial network in optimising transportation of goods has been established. The target function in the model includes: fixed prices for construction of big and small warehouses; variable prices for transportation of raw materials and goods between warehouses and commercial points. The algorithm was created for optimisation of local roads network between commercial points. As substantiated, if density of distribution of target function values in the zone of extreme is fairly high, the independent random search is sufficiently effective even when comparing it with regular methods.
\end{abstract}

Keywords: warehouses, commercial network, goods, transportation, price, road transport freight, the simulation procedure of optimizing.

\section{Introduction}

The current commercial giants, e.g. Hyper RIMI (or MAXIMA) have located their commercial points in various places of the country. They have been accumulating and producing certain goods in big warehouses, and storing other goods in warehouses located in certain points. Goods are being transported between the above points by road transport means of certain capacity. On the one hand these increase the flows of transport means by burdening the roads, on the other hand they pollute the environment with the exhaust gases and, moreover, their price increases due to irrational transportation of goods. Therefore it is necessary to optimise distribution of big and small warehouses within the network of these commercial giants. Thus, it is necessary to define the number and place of big and small warehouses the construction and transportation of which could incur the lowest total goods costs $(z)$. The argument that it is necessary to optimise distribution of warehouses within the commercial network, has been received via the synthesis of a typological structure of the regional system of the freight road transport, see researches by Baublys and Išoraite (2006), and Baublys (2003).

Having carried out the synthesis of a typological structure of the road transport freight regional system, it was substantiated that it is necessary to optimise distribution of warehouses within the network, as well as to optimise the local roads network between commercial points.

\section{Optimisation model}

Formally the task looks as follows:

It is necessary to minimise

$$
z=\sum_{i=1}^{l} f_{i} y_{i}+\sum_{j=1}^{m} g_{j} z_{j}+\sum_{i=1}^{l} \sum_{j=1}^{m} \sum_{k=1}^{n} c_{i j k} x_{i j k} \text {. }
$$

Under the following conditions

$$
\begin{aligned}
& \sum_{i=1}^{l} \sum_{j=1}^{m} x_{i j k}=1, k=1,2, \ldots, n, \\
& \sum_{j=1}^{m} x_{i j k} \leq y_{i}, i=1,2, \ldots, l ; k=1,2, \ldots, n, \\
& \sum_{j=1}^{m} x_{i j k} \leq y_{i}, j=1,2, \ldots, m ; k=1,2, \ldots, n, \\
& y_{1} \leq z_{i}, i=1,2, \ldots, l, \\
& y_{i}, z_{j} \in(0,1), x_{i j k} \geq 0, \\
& i=1,2, \ldots, l ; j=1,2, \ldots, m ; \quad k=1,2, \ldots, n,
\end{aligned}
$$

where $f_{i}$ - construction price of a warehouse in place $i$, beside which manufacturing of certain goods is executed; $g_{j}$ - construction price of a small warehouse in $j$ place; $c_{i j k}$ - price of delivery of goods to commercial point $k$ from a warehouse-manufacturer, located in place $i$ to a small warehouse located in point $j ; y_{i}-$ binary variable which is equal to 1 , in case if a warehouse-manufacturer is in place $i$, and equal to 0 in other case; $z_{j}$ - binary 
variable which is equal to 1 , in case if a warehouse is in station $j$, and equal to 0 in other case; $x_{i j k}$ - incessant variable which equals the part of demand in commercial point $k$, warehouse-manufacturer $i$ of supplied goods via warehouse $j$.

Target function (1) consists of a fixed construction price $f_{i}$ and $g_{j}$ correspondingly of warehouses-manufacturers and small warehouses, and variable prices $c_{i j k}$ (of raw-material and goods to be transported to a warehousemanufacturer $i$ ), transportation costs between stations $i$ and $j$ and transportation costs between warehouse $j$ and commercial point $k$. Equality (2) means that demand of each commercial point should be fulfilled. Terms (3) and (4) mean that demand of a commercial point $k$ can be fulfilled only for goods from the warehouse located in territory $i$, via a small warehouse located in territory $j$. Inequality (5) guarantees that a big warehouse is also a manufacturer, and term (6) - that construction of big and small warehouses has been completed, and that part of supplied goods is a plus.

In case if values of variables $y_{i}$ and $z_{j}$ in expressions (1)-(6) are known, optimal values of remaining variables are estimated in the following way: variable $x_{i j k}$ of each variable $k$ corresponds to the minimal $c_{i j k}$ value, in case if condition $y_{i}=z_{j}=1$ is fulfilled, and variables $x_{i j k}$ are considered as equal to 0 . Then the task $(1-6)$ can be solved by selecting vectors $Y=\left(y_{i}\right)$ and $Z=\left(z_{i}\right)$. Each of iteration respective variables is fixed 0 , others -1 , and some of them remain unfixed. Let

$$
\begin{aligned}
& K_{0}^{I}=\left\{i / y_{i}=0\right\}, \\
& K_{1}^{I}=\left\{i / y_{i}=1\right\}, \\
& K_{2}^{I}=\{1,2, \ldots, l\} \backslash K_{0}^{I} \cup K_{1}^{I}
\end{aligned}
$$

mean the set of variables $y_{i}$, having corresponding values 0,1 and free variables, and

$$
\begin{aligned}
& K_{0}^{I}=\left\{j / z_{j}=0\right\}, \\
& K_{1}^{I}=\left\{j / z_{j}=1\right\}, \\
& K_{2}^{I}=\{1,2, \ldots, m\} \mid K_{0}^{I} \cup K_{1}^{I}
\end{aligned}
$$

mean corresponding sets of variables $z_{j}$. After having changed fixed variables by their values in the formulas (1-6), we get the following task which has to be minimised:

$$
\begin{aligned}
& Z=\sum_{i \in K_{1}^{I}} f_{i}+\sum_{j \in K_{1}^{I}} g_{j}+\sum_{i \in K_{2}^{I}} f_{i} y_{i}+ \\
& \sum_{j \in K_{2}^{I}} g_{j} z_{j}+\sum_{i \in K_{1}^{I} \cup K_{2}^{I}} \sum_{j \in K_{1}^{I} \cup K_{2}^{I}} \sum_{k=1}^{n} c_{i j k} x_{i j k},
\end{aligned}
$$

under the following conditions,

$$
\begin{aligned}
& \sum_{i \in K_{1}^{I} \cup K_{2}^{I}} \sum_{j \in K_{1}^{I} \cup K_{2}^{I}} x_{i j k}=1 ; \quad k=1,2, \ldots, n, \\
& \sum_{j \in K_{1}^{I} \cup K_{2}^{I}} x_{i j k} \leq y_{i}, \quad i \in K_{2}^{I} ; \quad k=1,2, \ldots, n,
\end{aligned}
$$

$$
\begin{aligned}
& \sum_{i \in K_{1}^{I} \cup K_{2}^{I}} x_{i j k} \leq z_{j}, \quad j \in K_{2}^{I} ; \quad k=1,2, \ldots, n, \\
& y_{i} \leq z_{j}, \quad i \in K_{2}^{I}, \\
& y_{i}, z_{j} \in\{0,1\}, \quad i \in K_{2}^{I}, \quad j \in K_{2}^{I}, \\
& x_{i j k} \geq 0, \quad i \in K_{1}^{I} \cup K_{2}^{I}, \quad j \in K_{1}^{I} \cup K_{2}^{I} ; \\
& k=1,2, \ldots, n .
\end{aligned}
$$

Let $c_{m k}$ mean the minimal goods transportation price to $k$ commercial point from a big warehouse $i \in K_{1}^{I}$ via a small warehouse $j \in K_{1}^{I}$ :

$$
c_{m k}=\min _{i, j}\left\{c_{i j k} / i \in K_{1}^{I}, \quad j \in K_{1}^{I}\right\} .
$$

The main condition for the delivery of goods from the warehouse-manufacturer $i$ via the warehouse $j$ in case of any optimal answer (solution) is $c_{i j k} \leq c_{m k}$. It allows to get upper margins $m_{i}$ and $n_{j}$ of the number of commercial points, to which goods from warehouses $i$ and $j$ are delivered, i.e.

$$
\begin{aligned}
& m_{i}=\left|\left\{k \mid \exists j \in K_{1}^{I} \cup K_{2}^{I}: c_{i j k}<c_{m k}\right\}\right|, i \in K_{1}^{I}, \\
& n_{j}=\left|\left\{k \mid \exists i \in K_{1}^{I} \cup K_{2}^{I}: c_{i j k}<c_{m k}\right\}\right|, j \in K_{1}^{I} .
\end{aligned}
$$

Further $c_{i j k}$ to all $i \in K_{1}^{I} \cup K_{2}^{I}, \quad j \in K_{1}^{I} \cup K_{2}^{I}$ and $k=$ $1,2, \ldots, n$ is defined as follows:

$$
c_{i j k}^{\prime}=\left\{\begin{array}{l}
c_{i j k}+\frac{f_{i}}{m_{i}}+\frac{g_{j}}{n_{j}}, \quad i \in K_{2}^{I}, \quad j \in K_{2}^{I}, \\
c_{i j k}+\frac{f_{i}}{m_{i}}, \quad i \in K_{2}^{I}, \quad j \in K_{1}^{I}, \\
c_{i j k}+\frac{g_{j}}{n_{j}}, \quad i \in K_{1}^{I}, \quad j \in K_{2}^{I}, \\
c_{i j k}, \quad i \in K_{1}^{I}, \quad j \in K_{1}^{I} .
\end{array}\right.
$$

And $c_{r k}^{\prime}$ because of $k=1,2, \ldots, n$ is found from

$$
c_{r k}^{\prime}=\min _{i, j}\left\{c_{i j k} / i \in K_{1}^{I} \cup K_{2}^{I}\right\}, \quad j \in K_{1}^{I} \cup K_{2}^{I} \text {. }
$$

The lower margin of the answer of the task (13-19) is:

$$
Z^{\prime}=\sum_{i \in K_{1}^{I}} f_{i}+\sum_{j \in K_{1}^{I}} g_{j}+\sum_{k=1}^{n} c_{r k}^{\prime} \text {. }
$$

The value of optimal solution (13)-(19) cannot be higher than

$$
\sum_{i \in K_{1}^{I}} f_{i}+\sum_{j \in K_{1}^{I}} g_{j}+\sum_{k=1}^{n} c_{m k}
$$

If a warehouse-manufacturer is in point $i$, then value (26) will decrease (or increase) by values

$$
p_{i}=\left[\sum_{k=1}^{n} \max _{j \in K_{1}^{I} \cup K_{2}^{I}}\left(0, c_{m k}-c_{i j k}\right)\right]-f_{i} .
$$

If a warehouse is in point $j$, then value (26) will decrease (or increase) by value 


$$
g_{j}=\left[\sum_{k=1}^{n} \max _{i \in K_{1}^{I} \cup K_{2}^{I}}\left(0, c_{m k}-c_{i j k}\right)\right]-g_{j} .
$$

Since total (26) decrease due to a possible assumption on location of several big and small warehouses cannot exceed decreases of the sum, corresponding to the location of each big and small warehouse, the following result is valid: (13)-(19) the lower margin of solution is defined as follows:

$$
\begin{aligned}
& z^{\prime \prime}=\sum_{i \in K_{1}^{I}} f_{i}+\sum_{j \in K_{1}^{I}} g_{j}+\sum_{k=1}^{n} c_{m k}- \\
& \sum_{i \in K_{2}^{I}} \max \left(p_{i}, 0\right)-\sum_{j \in K_{2}^{I}} \max \left(g_{i}, 0\right) .
\end{aligned}
$$

Another algorithm, based on the method of branches and margins, was tested.

$1 s t$ block. Let $z_{\text {opt. }}$ be value of the best solution which corresponds at the beginning to a free big number.

2nd block. Th e first check for optimality. We esti mate values of $m_{i}, n_{j}, c_{i j k}^{\prime}$ and $c_{r k}^{\prime}$ according to (21)-(24). Find $z^{\prime}$ according to (25). If $z^{\prime} \geq z_{\text {opt. }}$, we proceed to 5 th block.

$3 r d$ block. Checking of solution. If variables $y_{i}$ and $z_{i}$ are fixed under 0 or 1 , then we fix the found answer, resume value $z_{\text {opt. }}$ and proceed to 8 th block.

4th block. Th e first conditional checking of optimal ity. We estimate values $p$ of all $i \in K_{1}^{I}$ according to (27). If $p_{i}<0, y_{i}=0$.

5th block. Th e second conditional checking of opti mality. We estimate $j \in K_{1}^{I} g_{j}$ of all according to (28). If $g_{j}<0, z_{j}=0$.

6th block. Th e second checking of optimality. We es timate $z^{\prime \prime}$ according to (29). If $z^{\prime \prime}>z_{\text {opt. }}$, then we proceed to 8th block.

7 th block. Selection of a move. If during checking at least one variable is fixed in 4th and 5th blocks, we proceed to 2nd block. Otherwise $p_{k}=\max _{i \in K_{2}^{I}} p_{i}, g_{l}=\max _{j \in K_{2}^{I}} g_{j}$. If $p_{K} \geq g_{\text {, }}$, then $y_{K}=1$, otherwise $y_{K}=0$. We proceed further to 2 nd block.

8th block. Regression. We will search for the last variable $y_{K}$ or $z_{l}$, fixed under 1 . If there is no such variable, estimation is finalised. Otherwise all free after $y_{K}$ or $z_{l}$ variables are equal to 0 ; we fix $y_{K}$ or $z_{l}$ under 0 and proceed to 2nd block. Algorithm ends after the finite number of steps. We return back to block (8) only when a variable is fixed or when value of a variable changes.

\section{Optimisation of local roads network between commercial points}

Th e given network under establishment with $m$ nonoriented branches and $n$ hubs. Th e initial information is presented in distribution density $g(X) s$-meter vector $X$ area $Y$. Components $X$ are lengths $z_{i}$ of limits $N$ and transport connections $p_{i j t}$ between hubs $(i, j)$ in pair years $t$ during the planning period $[Q, T]$.

Th e trajectory has to be found in which

$$
\int F(\gamma, X) g(X) d X \rightarrow \min _{\gamma \in G} .
$$

Total expenditure $F(f, X)$ - an additive (according to network branches) function, basically depending on branch loading vector $\bar{Q}(t)$, the components of which are

$$
Q_{l t}=\sum_{v=1}^{3} p_{i} \sum_{i, j=1}^{n} \alpha_{l i j t}^{v} P_{i j t},
$$

where $p_{v}$ - probability in accepting one or other routing criteria (distance, time of transportation, transport costs); $\alpha_{l i j t}^{v}$ - supplementary binary values, defined by a complex of tasks about the shortest road for each connection:

$$
\begin{aligned}
& \sum_{l=1}^{M} \alpha_{l i j t}^{v} r_{l}\left(\sigma_{1 v}+\frac{\sigma_{2 v}}{v\left(q_{l t}\right)}+b\left(q_{l t}\right) \sigma_{3 v}\right) \rightarrow \min , \\
& \sum_{l \in I_{m t}} \alpha_{l i j t}^{v} \equiv\left(\sigma_{i m}+\sigma_{j m}\right)(\bmod 2), \\
& \alpha_{l i j t}^{v} \geq 0
\end{aligned}
$$

where $v\left(q_{l t}\right), b\left(q_{l t}\right)$ - average speed of transportation and cost price, when technical level is $q_{l t}$;

$$
\sigma_{a b}=\left\{\begin{array}{l}
1, \text { when } a=b, \\
0, \text { when } a \neq b .
\end{array}\right.
$$

$I_{m t}$ - a set of branches identical to a hub $m$, in which traffi $\mathrm{c}$ is permissible in yeart.

Th e set of trajectories $G$, in which functional (1) is minimised, is predetermined by the following conditions:

$$
\begin{aligned}
& \bar{q}(0)=\bar{q}_{0}, \\
& \bar{q}(T)=\left\{\phi\left(Q_{1 T}\right), \ldots, \phi\left(Q_{M T}\right)\right\}, \\
& \left\{q_{l t} \neq q_{l 0}\right\} \rightarrow\left\{q_{l t} \geq \phi\left(Q_{l t}\right)\right\}, \\
& \{D(t) \neq \varnothing\} \rightarrow\{\bar{q}(t) \in D(t)<\bar{q}(T)\}, \\
& \sum_{l=1}^{M} R_{d t l} \leq R_{d t} .
\end{aligned}
$$

Th e above conditions:

(35) - define the initial point of a trajectory;

(36) - define the final point, in which design standards $f$, predetermining technical level by load, are sustained;

(37) - indicate that any reconstruction measure leads to technical level which is not lower than required by design standards;

(38) - define whether there are social restrictions: among them - restrictions of the state, reachable in year $t$, guarantee connection between all points of a region;

(39) - define whether there are any resource restrictions, defined for each resource $d$ for all years $t$.

When network is optimised, then technical level of $l$ branch in year $t$ can be conditionally defined as follows:

$$
q_{l t}=\frac{T-t}{T} q_{l 0}+\frac{t}{T} q_{l T}
$$


i.e. anticipate an even change of the technical level from the initial $q_{l 0}$ up to the final $q_{l T}$, which is a controllable variable.

The task of optimisation of the current network (this is the totality of branches, by which transport connections can be maintained at any moment) - the search for the status $\bar{q}(T)$ network $N$, which could minimise total expenditure:

$$
\begin{aligned}
& \sum_{l=1}^{M} r_{l}\left[f_{1}\left(q_{l 0}, q_{l T}\right)+f_{2}\left(q_{l 0}, q_{l T}\right) N_{l}(0)+\right. \\
& \left.f_{3}\left(q_{l 0}, q_{l T}\right) N_{l}(T)\right] \rightarrow \min _{\bar{\Delta}(T)} .
\end{aligned}
$$

Traffic intensity in branches $N_{l}(t)$ is defined by summing up the routed connections:

$$
N_{l}(T)=\sum_{i, j=1}^{n} \alpha_{l i j} p_{i j T} .
$$

Here binary routing indicators $\alpha$ are derived by solving a system of tasks about the shortest road:

$$
\begin{aligned}
& \sum_{l=1}^{M} \alpha_{l i j} r_{l} \rightarrow \min , \\
& \sum_{l \in I_{m T}} \alpha_{l i j} \equiv\left(\sigma_{i m}+\sigma_{j m}\right)(\bmod 2), \\
& \alpha_{l i j} \geq 0 .
\end{aligned}
$$

Here markings are the same as in (32)-(34). Technical level is found simply:

$$
\left(N_{i-1}<N_{l}(T) \leq N_{i}\right) \rightarrow\left(q_{l T}=i\right),
$$

where $N_{i-1}, N_{i}$ - technical level of the defined mark $i$ will be applied a priory.

In expression (41) functions $f_{1}, f_{2}, f_{3}$ evaluate all costs, depending and not depending on traffic, as well as their discounting. These functions do not depend on the decision and, therefore, can be estimated approximately in the form of value tables, and size of tables should not exceed $10 \times 10$. Of course, traffic intensity at the beginning $N_{t}(0)$, as well as the initial technical levels $q_{l 0}$ and lengths $r_{l}$ are known. Since the final state $\bar{q}(T)$ is definitely determined by the load vector $\bar{N}(T)$, and this vector also predetermines summation of sets $I_{m T}$, then binary vector $\bar{\Delta}(T)$ is a controlled variable:

$$
\delta_{l t}= \begin{cases}1, & q_{l t}>0 \\ 0, & q_{l T}=0 .\end{cases}
$$

It should be noted that it is impossible to functionally describe dependence of $\bar{N}(T)$ on $\bar{\Delta}(T)$, derived from (42)-(46).

Simulation procedure of optimizing (141-(46) in fact is discrete and multi-extreme.

When newly designing or developing the current network, the pair $\bar{q}(\tau)$ and $\bar{q}(\theta)$ of network $N$ has to be found (usually $\tau=s$ and $\theta=0$ ), minimising the costs:

$$
\begin{aligned}
& \sum_{l=1}^{M} r_{l}\left[f_{4}\left(q_{l 0}, q_{l \tau}, q_{l \theta}, q_{l T}\right)+\right. \\
& f_{5}\left(q_{l 0}, q_{l \tau}\right) N_{l}(0)+ \\
& f_{6}\left(q_{l 0}, q_{l \tau}, q_{l \theta}\right) N_{l}(\tau)+ \\
& f_{7}\left(q_{l \tau}, q_{l \theta}, q_{l T}\right) N_{l}(\theta)+ \\
& \left.f_{8}\left(q_{l \theta}, q_{l T}\right) N_{l}(T)\right] \rightarrow \min _{(q(\tau), q(\theta))} .
\end{aligned}
$$

Functions $f_{4}-f_{8}$ in principle do not differ from $f_{1}, f_{2}$, $f_{3}$ in the earlier presented task. $f_{4}, f_{6}, f_{7}$ can be split into constituents, depending on two arguments, therefore it is possible to use the same task table method.

Traffic intensities in branches $N_{l}(t)$ are defined by summarising routed connections:

$$
N_{l}(t)=\sum_{i, j=1}^{n} \alpha_{l i j t} P_{i j t,} t=\tau, \theta, T \text {. }
$$

Here routing indicators $a$ are found by solving a system of tasks about the shortest way (drive time).

$$
\begin{aligned}
& \sum_{l=1}^{M} \alpha_{l i j t} r_{l} \frac{1}{v\left(q_{l t}\right)} \rightarrow \min , \\
& \sum_{l \in I_{m t}} \alpha_{l i j t} \equiv\left(\sigma_{i m}+\sigma_{j m}\right)(\bmod 2),
\end{aligned}
$$

$\alpha_{l i j t} \geq 0$.

A set of possible solutions $(\bar{q}(\tau), \bar{q}(\theta))$ corresponds to resource restrictions:

$$
\begin{aligned}
& \sum_{l=1}^{m} R_{d}\left(q_{l 0}, q_{l \tau}\right) \leq R_{d \tau}^{\prime}, d=1,2, \ldots \\
& \sum_{l=1}^{M} R_{d}\left(q_{l \tau}, q_{l \theta}\right) \leq R^{\prime} d \theta, d=1,2, \ldots \\
& \left|\sum_{l=1}^{M}\left[R_{d}\left(q_{l \tau}, q_{l \theta}\right)-R_{d}\left(q_{l 0}, q_{l \tau}\right)\right]\right| \leq \varepsilon_{d}, d=1,2 \ldots
\end{aligned}
$$

Here index $d$ indicates that requirements are set for $d$ type resources. Referring to (54) it is stated that sudden jumps while using resources should be avoided. Besides, there are also social restrictions (38) on $t=\tau, \theta$.

The initial state in the network (35) is known, and in terms of final network state $\bar{q}(T)$,

$$
\delta(\bar{q}(T))=\bar{\Delta}(T)
$$

Technical level $q_{l t}=(t=\tau, \theta)$ in this task is controlled and related to branch load via the operator $f$ (design standards):

$$
\begin{aligned}
& q_{l T}=\phi\left(N_{l}(T)\right), \\
& \left\{q_{l t} \neq q_{l 0}\right\} \rightarrow\left\{q_{l t} \geq \phi\left(N_{l}(t)\right)\right\}, t=\tau, \theta .
\end{aligned}
$$

Differently from the current simulation procedure of optimizing, where a priory limits to applying technical level dominated, here the optimal technical level can 
be chosen (in terms of total expenditure). When a priory limits are often used in design practice, the task can be formulated incorrectly due to principled possibility in defining technical level and distribution of flows.

It is clear that target function value depends on controlled variables - state of network $\bar{q}(t)$ (via functions $f_{i}$ ), as well as on loads of network branches $\bar{N}(T)$. We will try to reach the change of the network function upon variation of one variable $q_{l}$. If instead of $q_{l t}^{\prime}$ we analyse $q_{l t}^{\prime}$, then, of course, values of functions $f_{i}$ will change saltatorily. Characteristics of functions $f_{i}$ provide for a priory defining the size of this jump. Besides, it is possible to form functions $f_{i}$, the values of which under arguments of integer numbers coincide with values $f_{i}$, non-intermittent within the entire setting area. However, the target function remains intermittent, because transport flows spread due to the change of the network state. And since re-distribution occurs pursuant to minimising condition (32), independent from the common target function (30) (exceptionally to road transport), the size of a certain jump cannot be specified a priory. The implication is that target function $F$ does not allow to execute its a priory determination of its continuity.

Let us remind, that if solution options are investigated according to Bernul scheme, then with probability $p$ it might be stated that after $R$ tests the best option depends on a certain part $X$ - the best of all possible options, and a possible ratio

$$
R=\frac{\log (1-p)}{\log (1-X)} .
$$

The essential moment of this method is definition of a needed value $X$, since it is clear that access to $X-$ the best option does not guarantee sufficient proximity to the absolute extreme. The difference of target function values might be very big. If distribution density of target function values in the extreme zone is big, independent random search is fairly effective, even if compared with regular methods. First of all, given the values $p$ and $X$, suitable for practical usage, the number of analysed options varies from 200 to 500 . Secondly, plenty of options are defined, which are close to global extreme, which in many cases can be interpreted as indetermination zone after later evaluation of incomprehensiveness of the initial information. Usually such a zone is found via multicritical optimisation by applying regular methods.

\section{Conclusions}

1. The model for distribution of warehouses in the commercial network was established by optimising goods transportation. The target function of the model consists of: fixed prices for construction of big and small warehouses; variable prices of transportation of raw materials and goods between commercial warehouses and commercial points. It was also tested and justified by the branch and margin method.

2. The algorithm was created with a view to optimising the local road network between commercial points. During the algorithm tests it was proved that if distribution density of target function values is rather high in the zone of extreme, an independent random search is fairly effective even if compared with regular methods. Firstly, the number of analysed options is 200-500. Secondly, quite many options which are close to global extreme, are clarified, which in many cases might be interpreted as an indetermination zone after having evaluated incomprehensive initial information. Usually such a zone is found via multi-critical optimisation by applying regular methods.

\section{References}

Baublys, A.; Išoraitè, M. 2006. Synthesis of the topological structure of road freight transport system, Transport 21(3): 160-164. ISSN 1648-4142.

Baublys, A. et al. 2003. Transportas: technologijos, ekonomika, aplinka, sveikata: monografija [Transport: technologies, economics, environment, health]. Vilnius: Technika. 